\title{
Estabilização física de solo laterítico arenoso utilizando resíduos cerâmicos
}

\author{
Physical stabilization of a sandy lateritic \\ soil using ceramic wastes
}

\begin{abstract}
Jairo Salim Pinheiro de Lima ${ }^{1}$, Gabriela Oliveira Vicente ${ }^{1}$, Luiza Perroni Fagundes ${ }^{1}$, Cássio Lázaro Barros Cabral ${ }^{1}$, Felipe Augusto da Cruz ${ }^{1}$, Mauro Mitsuuchi Tashima ${ }^{1}$
\end{abstract}

\footnotetext{
${ }^{1}$ Universidade Estadual Paulista -UNESP, Campus de Ilha Solteira, Departamento de Engenharia Civil, Grupo de Pesquisa em Materiais Alternativos de Construção, MAC, Alameda Bahia, 550, Ilha Solteira, São Paulo, Brasil.

e-mail: jairo.lima@unesp.br, gabriela_olivic@hotmail.com, fagundesluiza94@yahoo.com.br, cassiocabral@outlook.com, jcarinhatojr@gmail.com, feacruz@gmail.com,maumitta@hotmail.com
}

\section{RESUMO}

O crescimento exagerado das cidades trouxe consigo problemas de cunho ambiental, social e econômico, tornando modelos de desenvolvimento sustentável imperativos à humanidade e à preservação do meio ambiente. Neste sentido, cabe ressaltar a quantidade de resíduos provenientes da construção civil e de resíduos provenientes da indústria da cerâmica vermelha e o significativo crescimento de ambos nos últimos anos. Uma alternativa para estes resíduos é a sua utilização em camadas de pavimentos. O objetivo deste trabalho é analisar a possibilidade de utilização de resíduos de cerâmica vermelha no processo de estabilização física de um solo laterítico arenoso. Para isso, foram avaliadas três porcentagens de resíduos (0, 50 e 60\%, em massa) e também foi verificada a influência da energia de compactação na determinação do índice de suporte California (CBR). Os resultados obtidos mostraram que o uso de resíduos cerâmicos na estabilização de solos é uma prática viável e que, o incremento da energia de compactação pode melhorar significativamente o valor de CBR do solo, alcançando valores $50 \%$ superiores a amostra de solo sem adição de resíduo para as misturas com $60 \%$ de resíduo cerâmico.

Palavras-chave: estabilização de solos, reaproveitamento de resíduos, cerâmica vermelha.

\section{ABSTRACT}

The growth of the cities has brought with environmental, social and economic problems, doing an awareness of sustainable development essential for the survival of humanity and for the preservation of the environment. In this way, it is important to highlight the amount of waste from construction and waste from the red ceramic industry and the significant growth of both in the last years. An alternative to this waste is its use in layers of pavements. The aim of this paper is to assess the possibility of use red ceramic wastes in the stabilization of a sandy lateritic soil. For that, three different percentages of ceramic wastes $(0,50$ and $60 \%$, in mass) were assessed and the influence of compaction energy was verified by California Bearing ratio (CBR) test. The obtained results showed that the use of ceramic wastes is a viable practice in the soil stabilization and, the enhancement on the energy compaction can improve significantly the CBR, yielding increment of $50 \%$ for samples containing $60 \%$ of ceramic waste.

Keywords: soil stabilization, reuse of wastes, red ceramic. 


\section{INTRODUÇÃO}

Embora o Brasil apresente uma extensa malha rodoviária, a carência nesse setor é grande: existe a necessidade de construção de estradas vicinais, vias urbanas, etc já que são elas que possibilitam a interligação das rodovias principais e, consequentemente, facilitam o rápido escoamento de bens e produtos [1]. Além disso, a construção de pavimentos tradicionais, constituídos usualmente por brita bem graduada, são excessivamente onerosos e incompatíveis com a extensão da malha rodoviária brasileira [2]. Também é importante destacar que a construção de pavimentos requer um grande volume de materiais e serviços e que, nem sempre os que estão localizados próximos às obras apresentam boas características para serem utilizados.

Diante da realidade apresentada, pesquisadores desenvolveram alternativas mais viáveis para a execução e construção de pavimentos flexíveis introduzindo novos conceitos e aplicando materiais alternativos. Neste sentido, pode-se mencionar a metodologia desenvolvida por Nogami e Villibor onde se propõe o uso de solos arenosos finos lateríticos em base de pavimentos [3], base estabilizada granulometricamente com utilização de solos lateríticos e bases de solo-agregado executada com solos tropicais [4]. Do mesmo modo, o emprego de materiais alternativos que possam melhorar a qualidade de solos in natura e/ou diminuir o consumo de materiais "nobres" é uma técnica que pode trazer diversos benefícios para a sociedade. Neste contexto, podese tomar como exemplo os resíduos de construção e demolição que, além do benefício técnico, pode trazer como consequência um benefício ambiental já que um enorme volume de resíduo pode ser reaproveitado evitando o descarte inadequado do material. De acordo com Mobuss [6], 70\% dos resíduos provenientes da construção civil poderiam ser reciclados, gerando uma economia de aproximadamente $\mathrm{R} \$ 8$ bilhões.

A aplicação do conceito de sustentabilidade e de economia circular aumenta a busca de alternativas para o emprego e consequente valorização dos resíduos [7], destacando que a engenharia civil tem mostrado um enorme potencial para a reutilização de resíduos urbanos, industriais, agropecuários e, até mesmo reempregar os próprios resíduos da construção civil [8-9], como é o caso do resíduo de cerâmica vermelha [10].

O setor cerâmico no Brasil constitui um dos maiores conglomerados industriais do gênero no mundo, com participação no PIB brasileiro de quase 1,0\%. Parte importante desse setor, a indústria de cerâmica vermelha, também denominada cerâmica estrutural, envolve a produção de elementos estruturais, de vedação e de acabamento para a construção civil (telhas, blocos, tubos, lajotas e pisos) e responde por um faturamento de $\mathrm{R} \$ 18,0$ bilhões/ano EELA [11].

Diversos estudos vêm sendo realizados sobre o reaproveitamento de resíduos cerâmicos na melhoria do comportamento de solos. Misturas produzidas com cacos de telhas e solos lateríticos foram avaliadas e mostraram ganhos expressivos no valor do módulo de resiliência, chegando a atingir 288\% acima do módulo do agregado, com resultados que indicam ser possível a aplicação destas misturas em camadas de pavimento DIAS [12]. Segundo Abdou e Bernucci [13], as misturas de solo com resíduo cerâmico que foram utilizadas nas camadas de base e sub-base do pavimento apresentaram valor mínimo médio de Índice de Suporte Califórnia de $62 \%$, com expansão nula, atendendo os limites preconizados pela norma para utilização em vias de baixo volume de tráfego. Do mesmo modo, Redivo [14] analisou 3 solos de diferentes regiões de Santa Catarina, misturados com cacos de telhas e tijolos nas proporções 30:70, 50:50 e 70:30 e concluíram que a mistura solo-agregado pode ser utilizada em camadas de sub-base de pavimentos com baixo volume de tráfego e que a sua utilização pode gerar uma economia superior a $20 \%$ no custo final da estrutura.

$\mathrm{O}$ presente trabalho tem como objetivo analisar a viabilidade de estabilizar fisicamente o solo laterítico arenoso da Região de Ilha Solteira - SP (Noroeste do estado de São Paulo - divisa com o estado de Mato Grosso do Sul) através do uso de resíduos de cerâmica vermelha. Destacar que a região de Ilha Solteira está rodeada por indústrias produtoras de cerâmica vermelha e que, o reaproveitamento deste tipo de material pode trazer benefícios, econômicos, sociais e ambientais para a região.

\section{MATERIAIS E MÉTODOS}

\subsection{Materiais}

O solo utilizado nos ensaios foi coletado cidade de Ilha Solteira - SP, cujo ponto de coleta está localizado na latitude -20.42841788 e longitude -51.34189814 . A coleta do solo foi feita manualmente, sendo que a primeira camada de solo foi removida (aprox. $50 \mathrm{~cm}$ ) por apresentar matéria orgânica. Foram coletadas aproximadamente $800 \mathrm{~kg}$ de amostra deformada de solo. $\mathrm{O}$ solo foi destorroado, secado ao ar e passado na peneira \#10 (peneira normatizada pela ASTM) antes de ser armazenado.

O resíduo cerâmico foi doado por uma indústria cerâmica da cidade de Três Lagoas - MS (região de Ilha Solteira) e constituía-se de cacos de telhas cerâmicas. Para garantir a homogeneidade do material, foram co- 
letados $200 \mathrm{~kg}$ de resíduo. O resíduo cerâmico foi triturado através de um rolo compactador, sendo que o material retido na peneira \# $25 \mathrm{~mm}$ foi selecionado para a realização dos ensaios. Destacar que a porcentagem de material descartado foi inferior a $5 \%$.

\subsection{Métodos}

Foram realizados os seguintes ensaios para a caracterização do solo: análise granulométrica [15], limites de consistência [16-18], ensaio de compactação Proctor para as três energias de compactação: normal, intermediária e modificada [19] e ensaio de índice de suporte Califórnia (CBR) [20]. Além disso, o solo foi classificado utilizando os métodos de classificação TRB e MCT. Como a classificação TRB não é adequada para avaliar alguns tipos de solo de climas tropicais, optou-se por realizar a classificação MCT (miniatura, compactado, tropical). O ensaio mini-MCV (miniatura, compactado, valor) foi realizado de acordo com DNER ME 258/94 [21].

Para o resíduo cerâmico, realizou-se o ensaio granulométrico, tomando como base a NBR 6458 [22]. Devido a elevada porosidade do resíduo cerâmico, o mesmo foi imerso em água durante 24 horas antes da sua utilização na produção das misturas solo-resíduo cerâmico. As misturas de solo e resíduo cerâmico foram realizadas em diferentes proporções: 100:0, 50:50 e 40:60, em massa. Para cada mistura e para cada energia de compactação, foi determinada a umidade ótima e a massa específica seca máxima. Para a condição de massa específica seca máxima e umidade ótima, foi realizado o ensaio de CBR, sendo determinados os valores de CBR e os valores de expansão das amostras.

\section{RESULTADOS E DISCUSSÕES}

\subsection{Caracterização do solo}

Os limites de consistência do solo foram determinados experimentalmente, sendo obtido um limite de liquidez (LL) de $24 \%$ e um limite de plasticidade (LP) de $17 \%$ (umidade correspondente à passagem do estado semi-sólido para o plástico). $\mathrm{O}$ índice de plasticidade foi calculado pela diferença do limite de liquidez e o limite de plasticidade, obtendo-se o valor de 7\%, que o classifica como de plasticidade média. Além disso, foi realizada a distribuição granulométrica do solo, sendo apresentado na Figura 1.

Com os dados de caracterização do solo (limites de consistência, granulometria por peneiramento e sedimentação), o mesmo foi classificado como A-4 pelo método de classificação TRB (AASHTO). De acordo com essa classificação, o solo seria um solo siltoso não plástico ou moderadamente plástico e, não adequado para uso em pavimentação. Como o sistema TRB não é adequado para classificar solos evoluídos pedogeneticamente, foi realizado a classificação MCT. Para isso, as curvas de deformabilidade resultantes da compactação permitiram determinar o coeficiente c'. Com as curvas de compactação equivalente a 12 golpes, obteve-se o coeficiente d', $135,7 \mathrm{~kg} / \mathrm{m}^{3}$. Após a compactação as amostras foram imersas em água com uma extrusão de $10 \mathrm{~mm}$. A perda por imersão (Pi) foi igual a 90\%. O índice de laterização e’ obtido foi 1,01. Deste modo o solo foi classificado como um solo laterítico arenoso do tipo II (areia argilosa). A expansão e contração deste solo, segundo o método MCT, é baixa. O coeficiente de permeabilidade e de adsorção são baixos. Ainda segundo a metodologia MCT, esse solo pode ser utilizado em base, sub-base, reforço do subleito compactado, subleito compactado, aterro compactado e revestimento primário.

\subsection{Caracterização do resíduo cerâmico}

Na Figura 1 é apresentada a curva granulométrica do resíduo cerâmico e do solo utilizado na pesquisa. A partir desses dados, foi possível calcular o coeficiente de não-uniformidade (CU) do resíduo cerâmico obtendo-se um valor igual a 10,0 e um coeficiente de curvatura (Cc igual a 2,3). Neste sentido, pode-se concluir que o resíduo apresenta uma uniformidade média e é bem graduado. Em termos de estabilidade de pavimentos, a graduação dos resíduos é de extrema importância visto que partículas bem adensadas aumentam o contato entre si e consequentemente diminuem os vazios da mistura, gerando assim melhor integração entre o solo e o resíduo. 


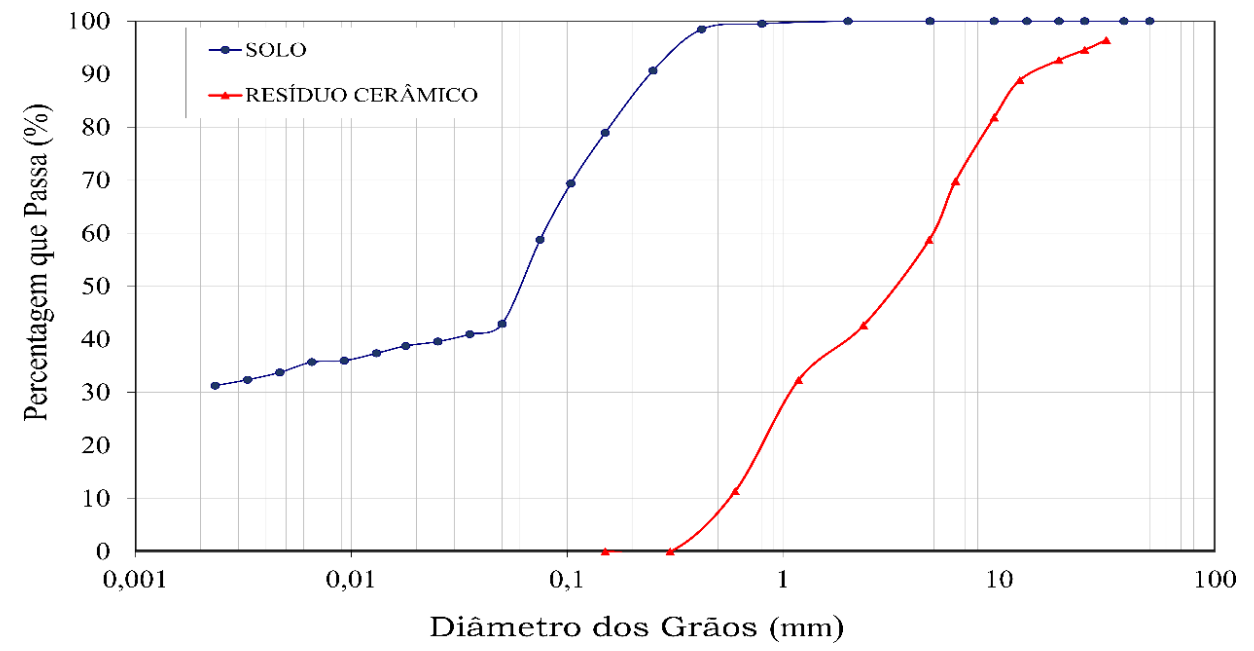

Figura 1: Curva granulométrica do solo e do resíduo cerâmico.

\subsection{Caracterização das misturas solo-resíduo cerâmico}

\subsubsection{Ensaio de Compactação}

A Figura 2 mostra as curvas de compactação para as diferentes porcentagens de resíduo cerâmico e para as diferentes energias de compactação utilizadas.

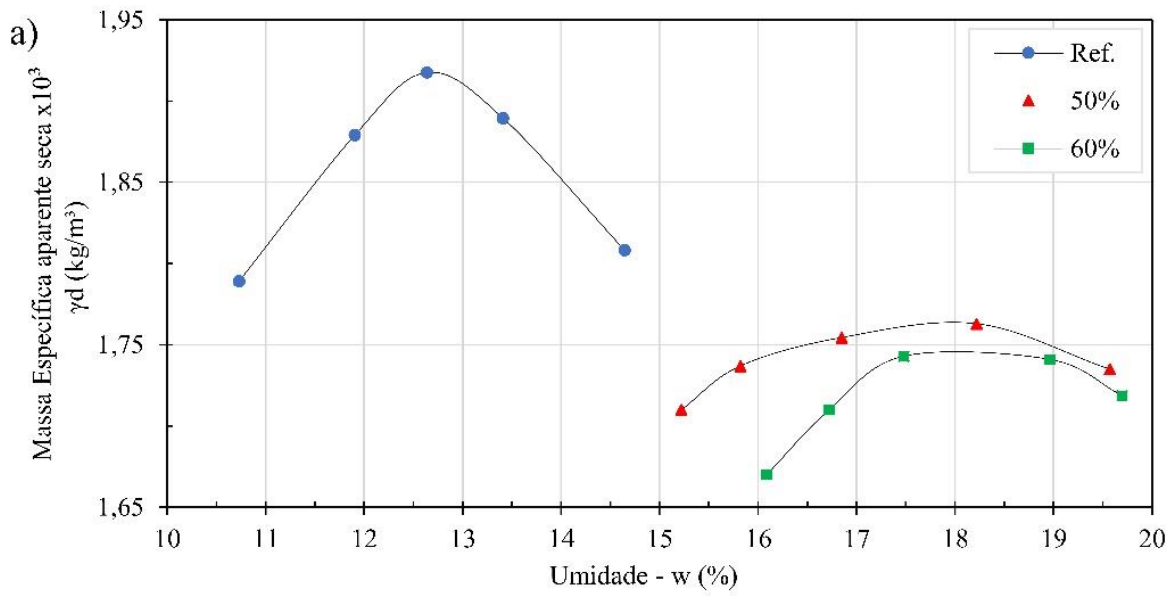



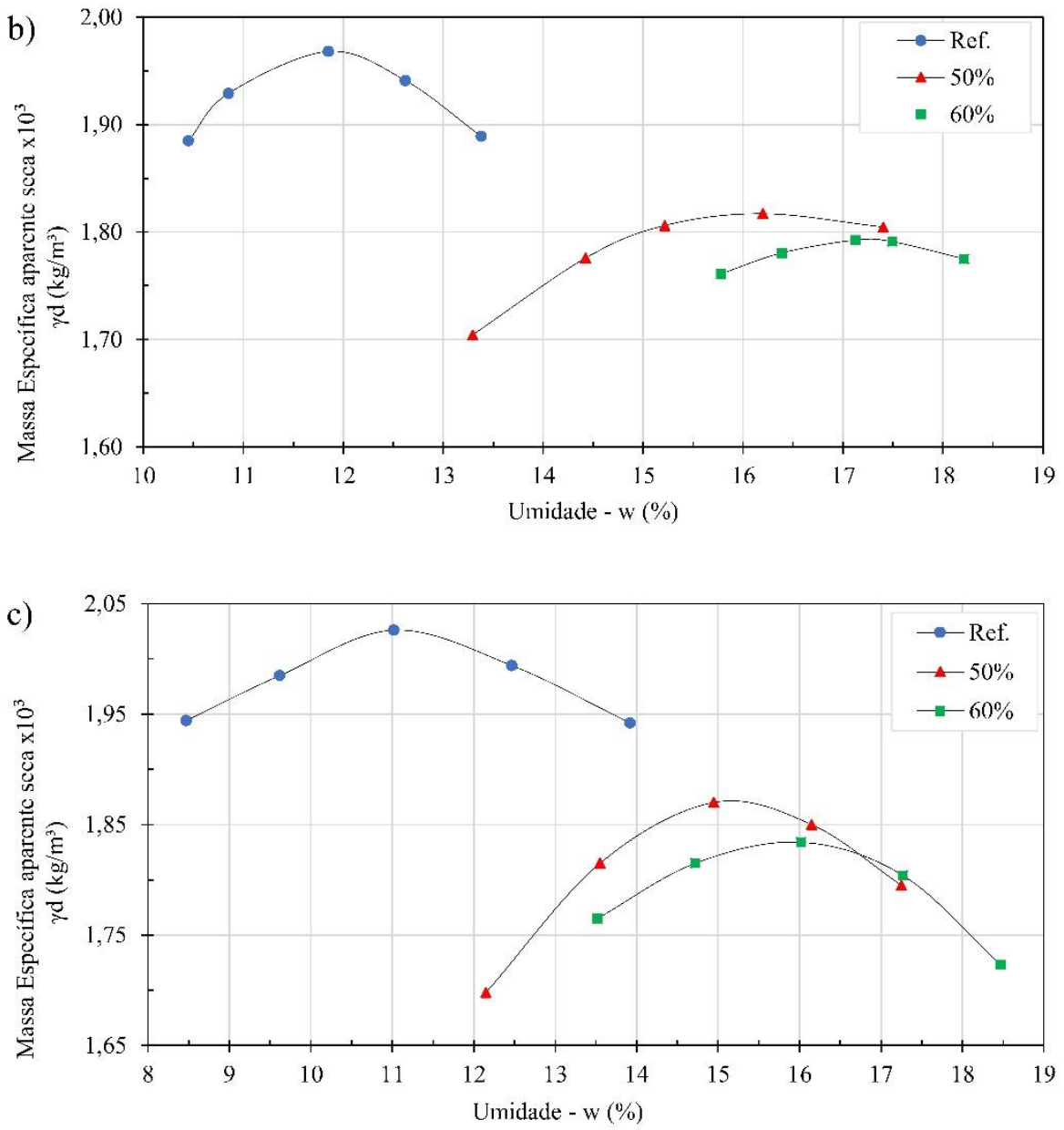

Figura 2: Curvas de compactação para diferentes percentuais de resíduo cerâmico: a) energia normal; b) energia intermediária; c) energia modificada.

O aumento da porcentagem de resíduo cerâmico teve como consequência uma diminuição nos valores de $\rho_{\text {dmáx. }}$ (massa específica aparente seca máxima). Isso ocorre já que a absorção de água pelo resíduo cerâmico é maior que a do solo, pois a porosidade do resíduo representa maior teor de umidade, além de menor valor de massa específica. As curvas de saturação das misturas de solo com resíduo cerâmico apresentam o mesmo comportamento para todas as energias aplicadas. Por apresentar características granulométricas diferentes das misturas, a curva de saturação do solo natural difere das demais, sendo deslocada para o ramo mais seco da saturação.

Ao analisar individualmente as amostras (Ref., 50 e 60\%) é possível notar que em todos os casos houve aumento da massa específica à medida que aumentava a energia aplicada às amostras. Isto é devido a redução no volume de vazios nas amostras. Proporcionalmente, quanto maior é a energia aplicada maior será sua a massa específica aparente seca (figura 3). Isso deve-se à diminuição dos vazios, pois a energia de compactação é inversamente proporcional ao índice de vazios. 


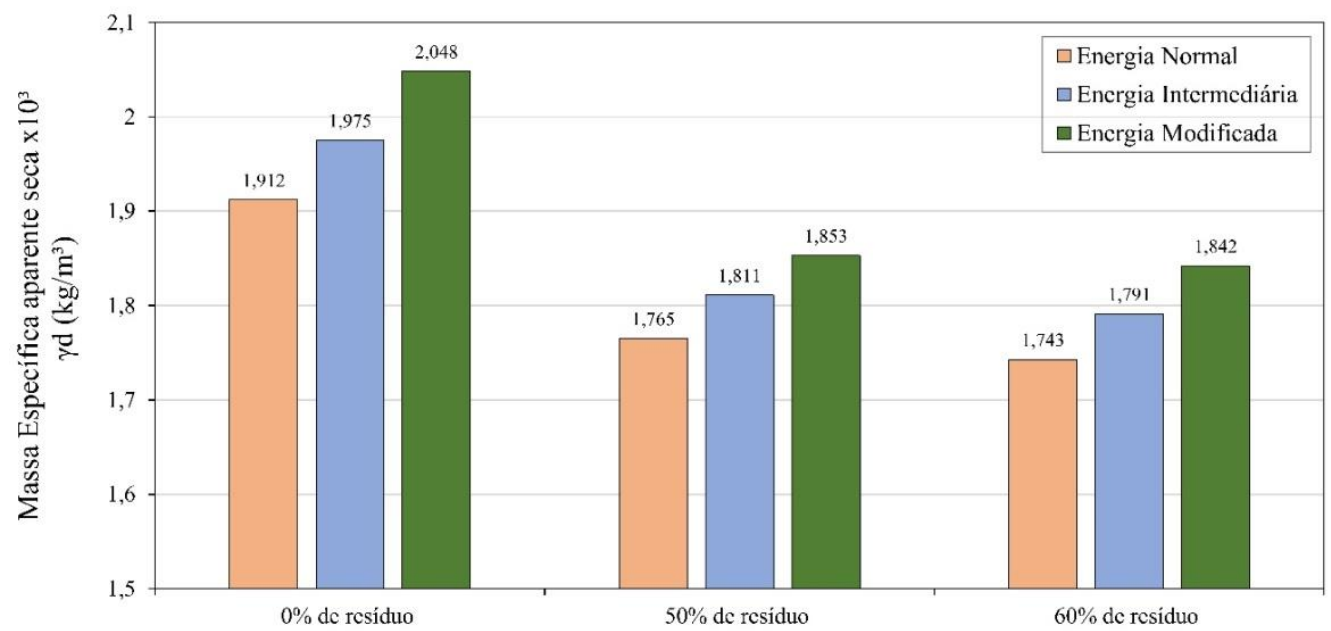

Figura 3: Comparação da $\rho_{\text {dmáx }}$ em função do percentual de resíduo para diferentes energias de compactação.

O aumento de energia de compactação também influenciou diretamente na definição da umidade ótima das misturas, visto que quanto maior é a energia de compactação, menor foi a quantidade de água necessária para obter a massa especifica aparente seca máxima, dado que a energia de compactação é inversamente proporcional ao índice de vazios da mistura, como pode ser visualizado na Tabela 1.

Tabela 1: Umidades ótimas das misturas em \%.

\begin{tabular}{c|c|c|c}
\hline \multirow{2}{*}{$\begin{array}{c}\% \\
\text { DUO RESÍ- }\end{array}$} & \multicolumn{2}{|c}{ ENERGIA } \\
\cline { 2 - 4 } & $\begin{array}{c}\text { NOR- } \\
\text { MAL }\end{array}$ & $\begin{array}{c}\text { INTERMEDI- } \\
\text { ÁRIA }\end{array}$ & MODIFICA- \\
\hline Ref & 12,64 & 11,85 & 11,02 \\
\hline 50 & 16,85 & 15,21 & 14,95 \\
\hline 60 & 17,48 & 17,13 & 16,02 \\
\hline
\end{tabular}

Com o acréscimo de resíduo cerâmico às amostras aumentou-se a umidade ótima das misturas devido a maior absorção da cerâmica, como visto anteriormente. Houve aumento de 33,31 e 38,29\% respectivamente para adição de 50 e $60 \%$ quando utilizada a energia normal na compactação em relação à umidade inicial sem adição de cerâmica. Para a energia intermediária, o aumento da umidade foi de 28,35 e 44,56\% para as amostras de 50 e $60 \%$ nesta ordem. Quando aplicada a energia modificada, este aumento foi na ordem de 35,66 e $45,37 \%$ para as amostras 50 e $60 \%$.

\subsubsection{Ensaio de CBR}

Para os valores de umidade ótima $\left(\mathrm{W}_{\text {ótm }}\right)$ e massa específica aparente seca máxima $\left(\rho d_{\text {máx }}\right)$ das energias de Proctor Normal, Proctor Intermediário e Proctor Modificado foram moldados corpos de prova que foram submetidos a ensaio de CBR. Os valores de CBR foram calculados para as penetrações em 0,1 e 0,2 polegadas $(2,54$ e 5,08mm), utilizando-se o maior valor dentre eles. $\mathrm{O}$ valor foi obtido da relação entre a pressão calculada (ou corrigida) sobre a pressão padrão, dado em porcentagem. A Figura 4 ilustra os resultados obtidos para o CBR das amostras compactadas para diferentes energias e proporções de resíduo. 


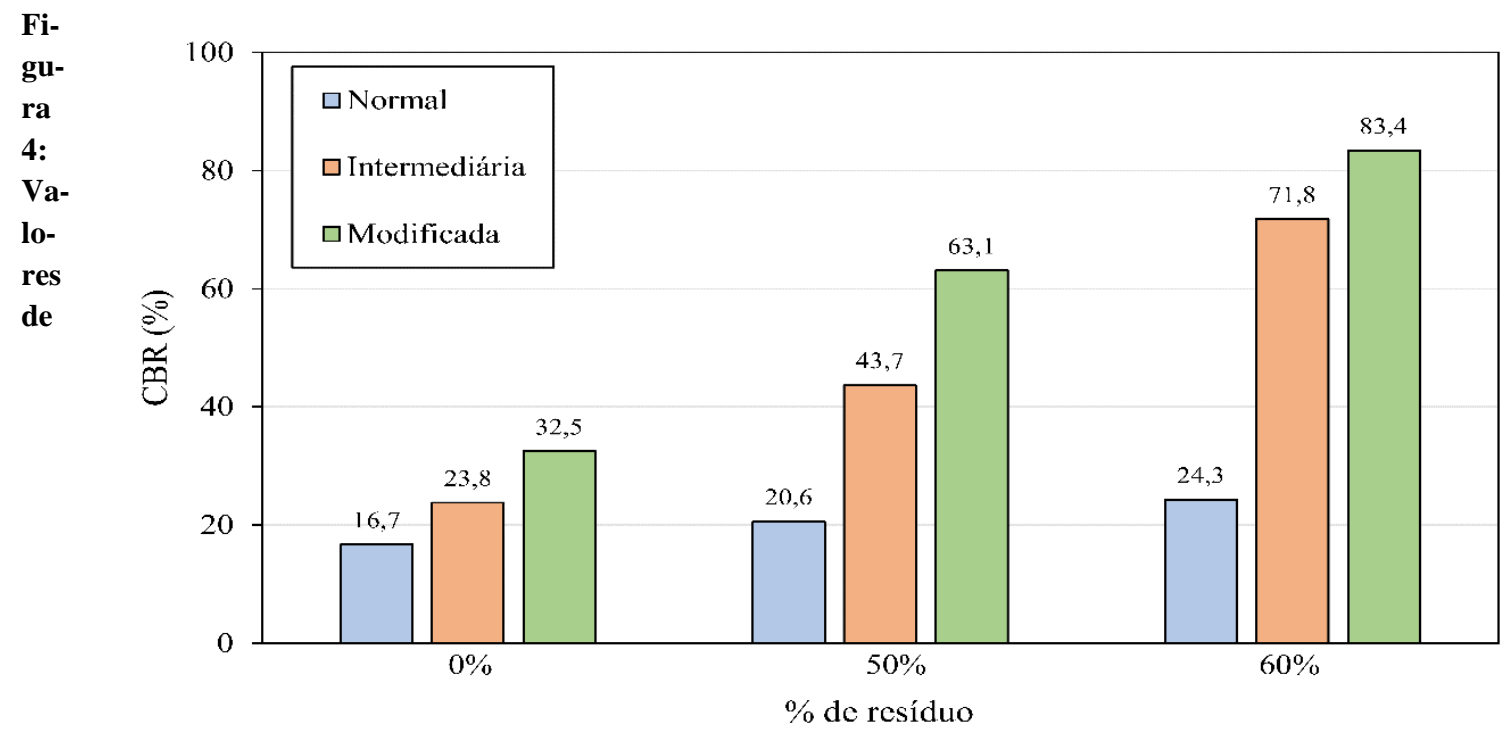

CBR para as diferentes misturas solo-resíduo cerâmico.

À medida que aumenta a energia de compactação, consequentemente os valores de CBR também se elevam. Para a amostra sem adição de resíduo o ganho foi de $42,51 \%$ para a energia intermediária, e $94,61 \%$ para a modificada em relação à energia normal.

Os cps com 50\% de resíduo apresentaram um aumento no valor do CBR foi de 112,14\% para a energia intermediária, e 206,31\% para a energia modificada, ambas em relação à energia normal.

A maior aumento no valor do CBR foi obtido com os cps com $60 \%$ de resíduo. Neste caso, o incremento nos valores foi de 195,47\% para energia intermediária, e 243,21\% para a energia modificada em relação à energia normal.

Além do CBR foi determinado também a expansibilidade do solo. Como pode ser observado na Tabela 1, para todas as amostras com adição de resíduo cerâmico houve uma redução na expansibilidade da amostra, quando comparado com a sua respectiva referência. Cabe ressaltar que mesmo apresentando pequenos valores de expansão, o DNIT permite aproveitar o solo laterítico como material para pavimentação rodoviária.

\begin{tabular}{l|l|l|l}
\hline \multirow{2}{*}{$\%$ Resíduo } & \multicolumn{2}{|c}{ Energia } & \multicolumn{2}{|c}{ Modificada } \\
\cline { 2 - 4 } & Normal & Intermediária & 0,1 \\
\hline $0 \%$ & 0,2 & 0,1 & 0,0 \\
\hline $50 \%$ & 0,0 & 0,0 & 0,0 \\
\hline $60 \%$ & 0,0 & 0,0 & \\
\hline
\end{tabular}

Tabela 2: Expansão das amostras (\%) em função das proporções de resíduos para diferentes energias de compactação.

Com os valores de CBR e expansibilidade do solo, pode-se utilizar a especificações do DNIT sobre o uso de solos estabilizados granulometricamente para pavimentos (ver Tabela 3). Para facilitar a discussão dos dados, a análise foi realizada para diferentes porcentagens de resíduo cerâmico e uma mesma energia de compactação. 


\begin{tabular}{l|l|l}
\hline Camadas & CBR $(\%)$ & Expansão $(\%)$ \\
\hline Sub-Base & $\geq 20$ & $\leq 1,0$ \\
\hline \multirow{2}{*}{ Base } & $>60$ para $\mathrm{N}<5 \times 10^{6}$ & $\leq 0,5$ \\
\cline { 2 - 2 } & $\geq 80$ para $\mathrm{N}>5 \times 10^{6}$ & \\
\hline
\end{tabular}

Tabela 3: Especificação de utilização do solo, adaptado DNIT [24].

Na Figura 5 destaca-se que o solo compactado na energia normal, com o acréscimo de resíduo a mistura solo-cerâmica passa de não-recomendado o uso na pavimentação (CBR < 20), para recomendado como subbase, tanto em misturas com 50\% quanto na mistura com 60\% de resíduo cerâmico reciclado. As Figuras 6 e 7 reproduzem os mesmos dados, porém para as energias de compactação intermediária e modificada, respectivamente.

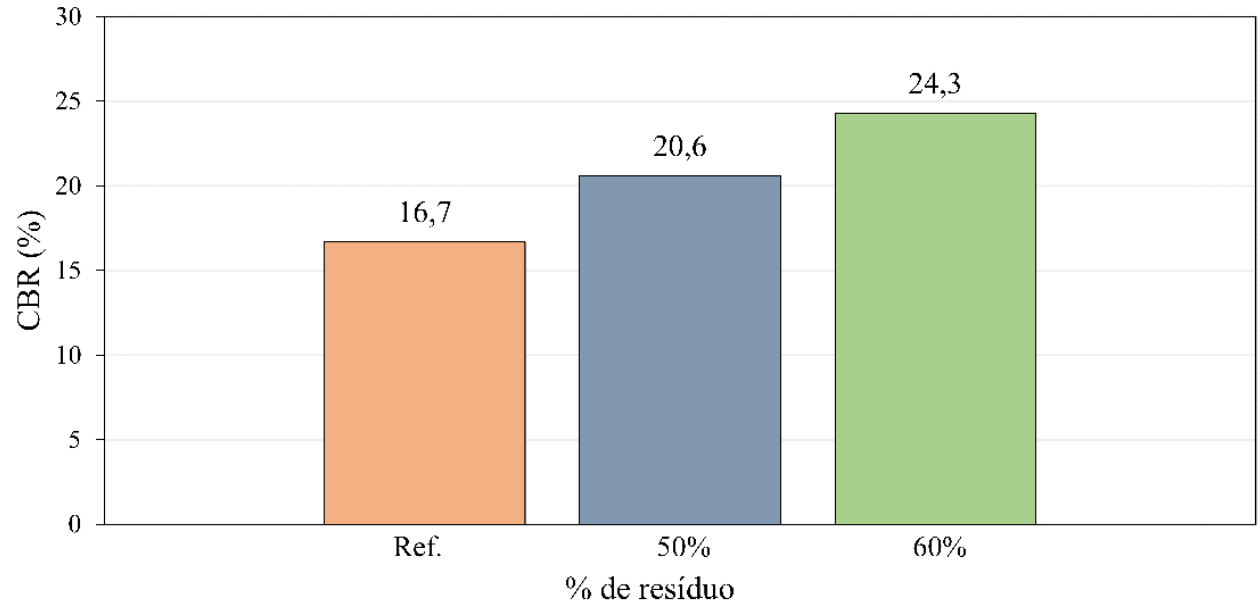

Figura 5: Os valores de CBR obtidos para energia do Proctor Normal para os percentuais de resíduo analisados.

Para as amostras compactadas com a energia intermediária (Figura 6) pôde-se observar que antes da estabilização granulométrica com o resíduo, o solo já era indicado para uso na pavimentação como sub-base.

Com a adição do resíduo cerâmico, a melhoria é significativa, quase chegando a dobrar o valor de capacidade de suporte do solo na mistura de $50 \%$ de resíduo, mas ainda mantendo a recomendação para uso como sub-base. Na mistura com $60 \%$ de resíduo cerâmico reciclado, a melhoria é ainda mais significativa, o solo passa de recomendável para uso como sub-base, para recomendável no uso como base, em tráfegos mais leves. 


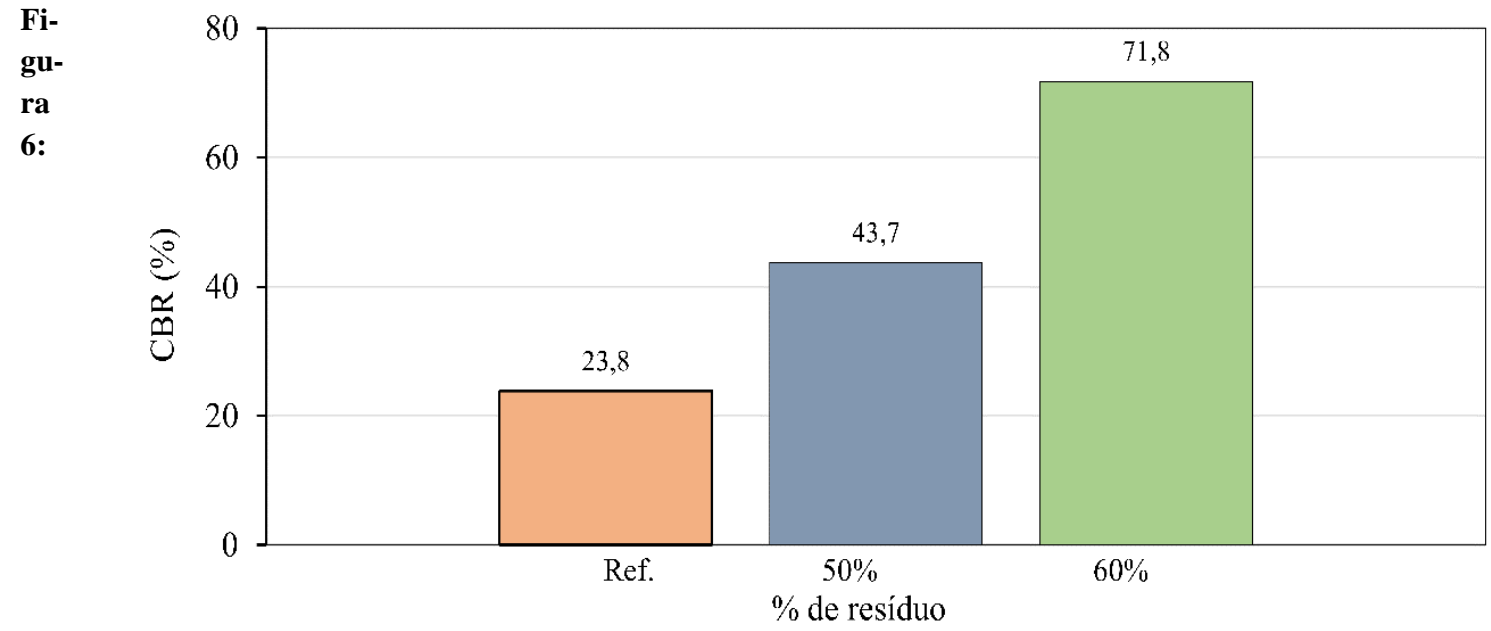

CBR para energia Intermediária.

Nas amostras compactadas com energia modificada (Figura 7), todos os teores proporcionaram valores elevados de CBR em relação às outras amostras, sendo o solo sem adição recomendado para utilização em sub-base, e para as demais amostras, com 50 e $60 \%$, podem ser utilizados em camadas de base.

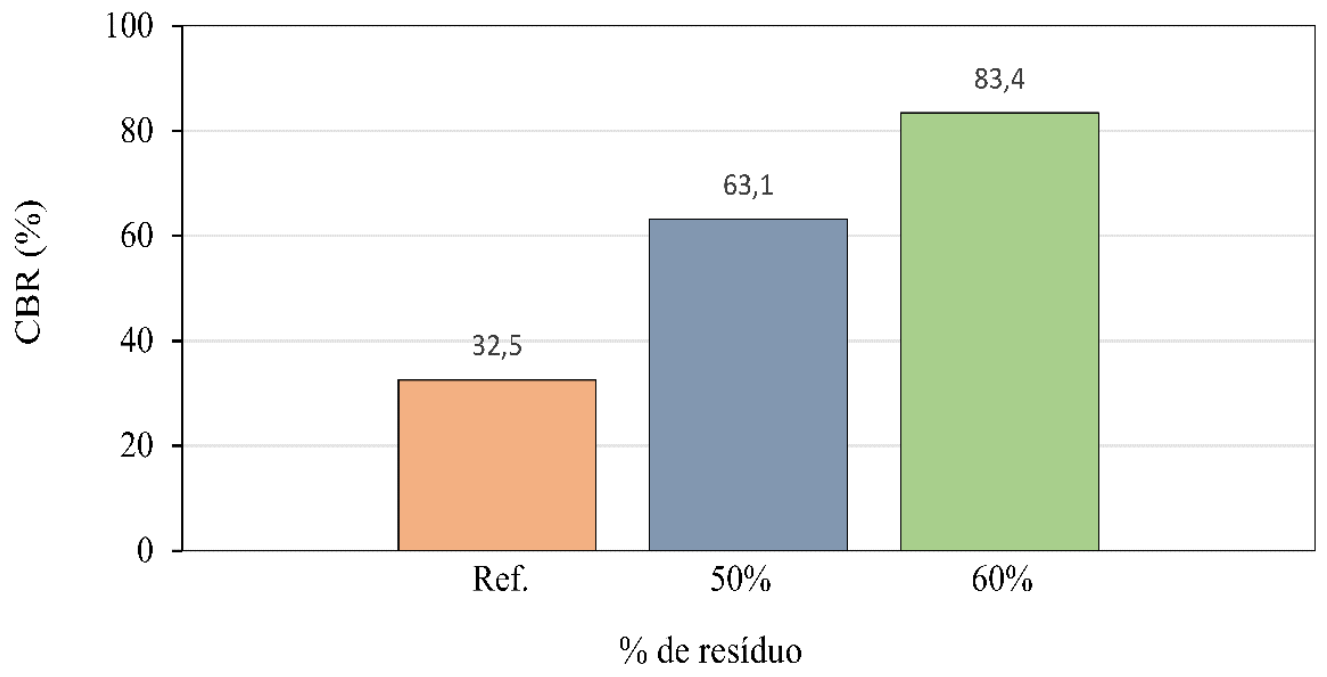

Figura 7: CBR para energia Modificada.

Em um estudo realizado por Abdou e Bernucci [13] foram alcançados valores de CBR de 62 e $75 \%$ para solo estabilizados com resíduos de construção e demolição utilizando energia intermediária. Estes valores são similares aos encontrados neste trabalho, para porcentagens de $50 \%$ de resíduo cerâmico.

\section{CONCLUSÕES}

Levando em consideração os resultados obtidos, pode-se obter as seguintes conclusões:

- A reutilização de resíduos cerâmicos mostrou-se vantajosa desde o ponto de vista meio ambiental, visto que grande parte deste tipo de resíduo não possui um descarte adequado.

- O emprego de resíduos cerâmicos na estabilização do solo laterítico arenoso mostrou-se uma técnica viável para utilização em pavimentos já que confere um aumento significativo na capacidade de suporte do solo viabilizando a sua utilização na pavimentação.

- Os valores de expansibilidade do solo foram reduzidos ou mesmo anulados com o uso dos resíduos ce- 
râmicos, independentemente da energia de compactação aplicada.

- Seguindo os critérios estabelecidos pelo DNIT, os solos com adição de resíduos cerâmicos poderiam ser utilizados perfeitamente como sub-base ou mesmo como base de pavimento, dependendo da energia de compactação utilizada.

\section{AGRADECIMENTOS}

Os autores gostariam de agradecer a empresa Cerâmica Guerra, localizada na cidade de Três Lagoas-MS pela doação do material utilizado nesta pesquisa.

\section{BIBLIOGRAFIA}

[1] CONFEDERAÇÃO NACIONAL DO TRANSPORTE. Boletim Estatístico - CNT -janeiro 2018. Disponível em: http://www.cnt.org.br/Boletim/boletim-estatistico-cnt

[2] VILLIBOR, D.F., et al., Pavimentos de Baixo Custo para Vias Urbanas: Bases alternativas com solos lateríticos Gestão de Manutenção de Vias Urbanas. 3. ed. São Paulo: Arte \& Ciência, 2009.

[3] NOGAMI, J.; VILLIBOR, D.F. Pavimentos Econômicos: Tecnologia do uso dos Solos Finos Lateríticos. São Paulo: Arte \& Ciência, p. 292, 2009.

[4] NOGAMI, J.; VILLIBOR, D.F. Uma nova classificação para finalidades rodoviárias. In: Simpósio Brasileiro de Solos Tropicais em Engenharia. Rio de Janeiro. Anais. Rio de Janeiro, CO-PE/UFRJ, p.30-41, 1981.

[5] BARROS, C.T. Propostas de novos critérios para especificação de bases de solo-agregado executadas com solos tropicais. 213p. Tese (Doutorado) - Escola politécnica, Universidade de São Paulo. São Paulo, 1989.

[6] MOBUSS CONSTRUÇÃO. Entenda a importância de um plano de gerenciamento de resíduos da construção civil. Disponível em: http://www.joaquimnabuco.edu.br/noticias/aprenda-fazer-os-principais-tipos-dereferencias-bibliograficas, 2017.

[7] PAYÀ, J., et al., Increasing the sustainability of alkali-activated binders: the use of sugar cane straw ash (SCSA). Construction and Building Materials. Espanha, v. 124, pp. 148-154, 2016.

[8] PRASARA-A, J.; GHEEWALA, S.H. Sustainable utilization of rice husk ash from power plants: A review. Journal of Cleaner Production. Thailand, v.167, pp. 1020 -1028, 2017.

[9] KREJCIRIKOVA, B., OTTOSEN, L.M., KIRKELUND, G.M., et al., Characterization of sewage sludge ash and its effect on moisture physics of mortar.

[10] SIDDIQUE, S., SANDEEP, C., SANDEEP, S., et. al. Sustainable utilisation of ceramic waste in concrete: Exposure to adverse conditions. Journal of Cleaner Production, India, v. 210, pp. 246-255, 2019.

[11] EELA. Panorama da indústria cerâmica vermelha no Brasil. Rio de Janeiro: [s.n.]. 2012.

[12] DIAS, J.F. Avaliação de resíduos da fabricação de telhas cerâmicas para emprego em camadas de pavimento de baixo custo.251p. Tese (Doutorado) - Escola Politécnica, Universidade de São Paulo. São Paulo, 2004.

[13] ABDOU, M. R., BERNUCCI, L.L.B. Pavimento ecológico: uma opção para a pavimentação de vias das grandes cidades. Escola Politécnica, Universidade de São Paulo. São Paulo, 2007.

[14] REDIVO, I. M. Utilização de resíduo de cerâmica vermelha em misturas com solo para construção de camadas de pavimentos com baixo volume de tráfego. 160p. Dissertação (Mestrado) - Universidade Federal de Santa Catarina. Florianópolis, 2011.

[15] ASSOCIAÇÃO BRASILEIRA DE NORMAS TÉCNICAS. NBR 7181: Solo - Análise Granulométrica. Rio de Janeiro: ABNT: 2016.

[16] ASSOCIAÇÃO BRASILEIRA DE NORMAS TÉCNICAS. NBR 6459: Solo - Determinação do Limite de Liquidez. Rio de Janeiro: ABNT: 1984.

[17] ASSOCIAÇÃO BRASILEIRA DE NORMAS TÉCNICAS. NBR 7180: Solo - Determinação do Limite de Plasticidade. Rio de Janeiro: ABNT: 2016.

[18] ASSOCIAÇÃO BRASILEIRA DE NORMAS TÉCNICAS. NBR 6457: Amostras de Solo - Preparação para ensaios de compactação e ensaios de caracterização. Rio de Janeiro: ABNT: 1986.

[19] ASSOCIAÇÃO BRASILEIRA DE NORMAS TÉCNICAS. NBR 7182: Solo - Ensaio de compactação. Rio de Janeiro: ABNT: 1986 
[20] ASSOCIAÇÃO BRASILEIRA DE NORMAS TÉCNICAS. NBR 9895: Solo - Índice de Suporte Califórnia. Rio de Janeiro: ABNT: 1987.

[21] DEPARTAMENTO NACIONAL DE ESTRADAS DE RODAGEM. ME 258/94: Solos compactados em equipamento miniatura. Rio de Janeiro: DNER, 1994.

[22] ASSOCIAÇÃO BRASILEIRA DE NORMAS TÉCNICAS. NBR 6458: Grãos de pedregulho retidos na peneira de $4,8 \mathrm{~mm}$. Determinação da massa específica aparente e da absorção de água. Rio de Janeiro: ABNT: 1984.

[23] ASSOCIAÇÃO BRASILEIRA DE NORMAS TÉCNICAS. NBR 15310: Componentes cerâmicos Telhas - Terminologia, requisitos e métodos de ensaio. Rio de Janeiro: ABNT: 2009.

[24] DEPARTAMENTO NACIONAL DE INFRAESTRUTURA DE TRANSPORTES 141/2010 - ES: Pavimentação - Base estabilizada granulometricamente - Especificação de serviço. Rio de Janeiro: IPR.

\section{ORCID}

Jairo Salim Pinheiro de Lima Gabriela Oliveira Vicente Luiza Perroni Fagundes Cássio Lázaro Barros Cabral Felipe Augusto da Cruz Mauro Mitsuuchi Tashima https://orcid.org/0000-0001-5482-8119 https://orcid.org/0000-0002-3494-7353 https://orcid.org/0000-0001-5011-4012 https://orcid.org/0000-0002-1091-5787 https://orcid.org/0000-0001-5394-8653 https://orcid.org/0000-0003-0885-9293 